\title{
OP - 0163: Peer support and education to better thrive with diabetes
}

\section{A. Norton, MS; N. Bereolos, PhD, MPH, CDE; C.M. Aprigliano, MS}
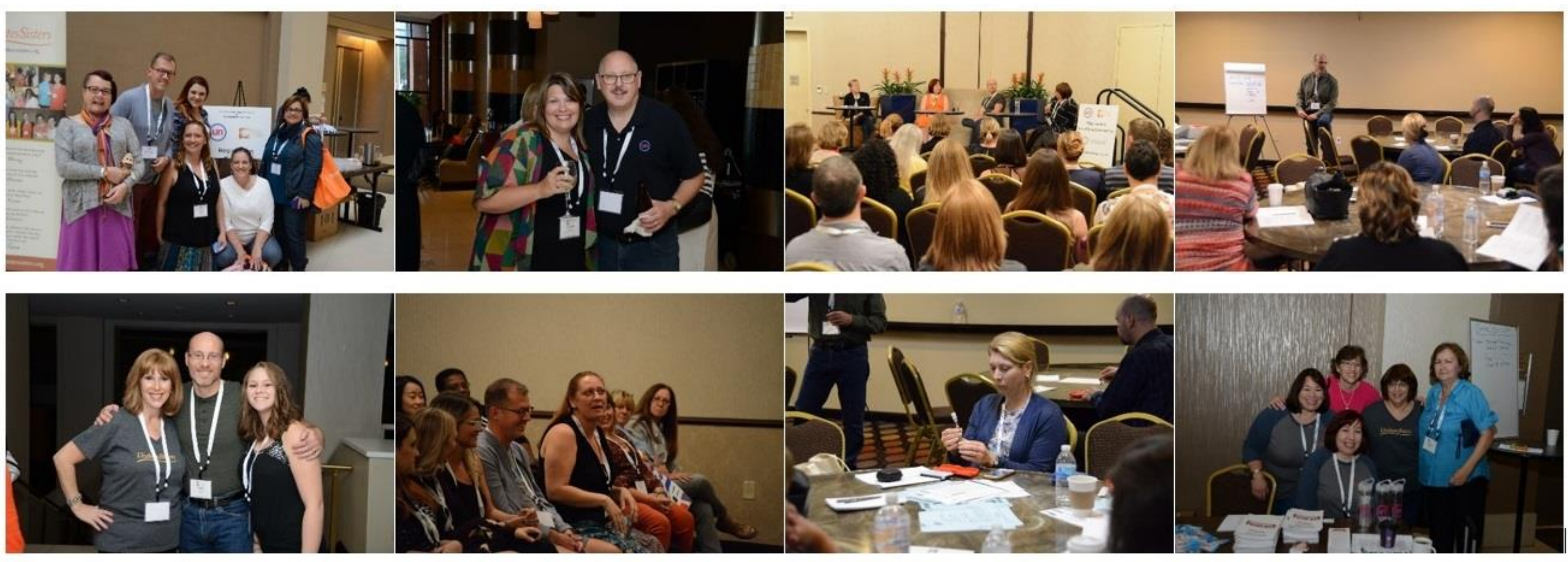

Background: While online communities are one source of support for people living with diabetes, in-person gatherings can offer psychosocial support, practical assistance, resources, and education from peers and professionals in a safe, non-judgment zone. The Diabetes UnConference (DUnCon) and Weekend for Women Conference (WfW), offered by The Diabetes Collective and DiabetesSisters respectively, offer facilitated peer support sessions from adults living with diabetes, along with formal presentations by professionals including endocrinology, psychology, cardiology, and nutrition, which provide the foundation for the knowledge shared.

Since 2010, DiabetesSisters, a non-profit patient-led organization providing support and education to women with diabetes, has hosted the WfW Conference, serving over 1,000 people. The two-day conference has converged across the United States to bring education, support and empowerment to women as they walk their journey with diabetes. In 2015, The Diabetes Collective, Inc., hosted its first DUnCon, an event where attendees provide diabetes topics they wish to discuss in an unscripted environment. The conference has alumni of 300+ people. In October 2017, these two organizations co-located their programs outside Washington, D.C. to bring people living with diabetes (and their loved ones) to experience a weekend of support and education.

Aims: Designed with respect and trust, both events promote inclusion of all kinds of diabetes, stressing that similarities and concerns far outweigh differences. Conversations among people living with diabetes included:

\begin{tabular}{ll} 
Guilt & Stigma \\
Challenges with Food & Fear \\
\hline Intimacy & Communication \\
Successes & Barriers \\
Preferred Use of Language & Psychological Burnout
\end{tabular}

Additionally, sessions led by content experts are invited to speak on topics such as:

$\begin{array}{ll}\text { Nutrition } & \text { Mental Health } \\ \text { Co-Occurring Condition such } & \text { Communicating with } \\ \text { as pregnancy, eating disorders, } & \text { Healthcare Providers } \\ \text { and heart disease } & \end{array}$

\section{Discussion from Attendees:}

"The connections with other people with diabetes \& the well-thought out educational sessions were helpful in my journey with diabetes. I enjoyed the community \& hearing from others who have been living with diabetes for $50+$ years.'

"I enjoyed the supportive \& friendly atmosphere, meeting so many others with diabetes, the information I received in sessions as well as informally by speaking with others, getting to meet the leaders \& staff of DiabetesSisters \& the Diabetes UnConference, the opportunities \& resources that were shared, \& the social events."

"I LOVED hanging out with my peers \& learning from them in all kinds of ways... be it exercise \& dancing, DIY pumps. It was empowering \& inspiring."

"I thought I was the only one who worried about my partner. I always wanted to help, I just didn't know how."
Results: Upon conclusion, attendees said they feel "empowered" and "inspired." Some have committed to seeking out different healthcare providers, while others have changed their perspective about using diabetes-related technology. Most departed the event with a new sense of community, a new tribe to consider family, and support to help them as they move forward in their journey. Others have created regional gatherings/monthly meetup groups to continue these conversations. Others have embraced "accountability" and "check in" partners to provide encouragement via text, email and/or social media. All attendees who responded to a post-event survey said they would recommend/highly recommend the weekend to others.

Branching out to those who support people with diabetes (partners/type $3 \mathrm{~s}$ ), these events provide conversations for many who have never openly verbalized their feelings. As with those living with diabetes, the partner may also lack peer support and have feelings of fear and being overwhelmed in how diabetes has impacted their lives. WfW and DUnCon offer "partner" tracks to better prepare them on what diabetes is (and isn't), how to communicate effectively, and how to handle emergencies. These sessions are also offered in a safe space, with no judgment.

For healthcare providers, it is challenging to cover the psychosocial aspects of living with diabetes in time-limited office visits. Events such as these allow for better living with diabetes between healthcare visits, empower patients to more proactively manage their condition, and have a more active involvement in treatment decisions.

These models of increasing empowerment via peer support can be translated across cultures and chronic conditions. It is a cost-containing method to improve the QoL. As the burdens of living with a chronic condition continues to intensify, the need for more holistic-care, outside of the traditional healthcare system, needs to be considered (Peyrot et al, 2013).

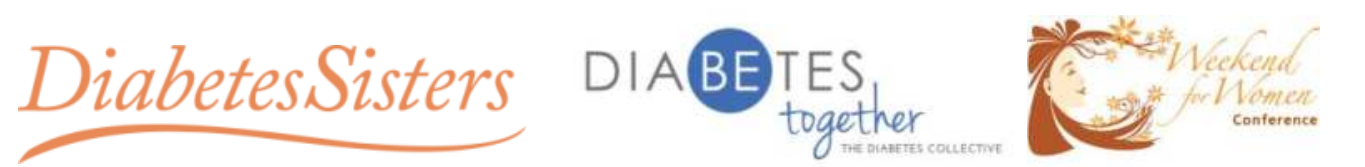

